\title{
OCEAN COLOR RETRIEVAL USING LANDSAT-8 IMAGERY IN COASTAL CASE 2 WATERS (CASE STUDY PERSIAN AND OMAN GULF)
}

\author{
N .Moradi a ${ }^{a}$ M. Hasanlou ${ }^{a} *$, M Saadatseresht $^{\text {a }}$ \\ ${ }^{a}$ School of Surveying and Geospatial Engineering, College of Engineering, University of Tehran, Tehran, Iran \\ (moradi.n, hasanlou, msaadat)@ut.ac.ir
}

Commission VIII, WG VIII/9

KEY WORDS: Ocean color, Landsat-8, MODIS, Visible and near infrared bands, Non-Linear Model

\begin{abstract}
:
Ocean color (OC) monitoring using satellite imageries provides an appropriate tool for a better understanding of marine processes and changes in the coastal environment. Radiance measurements in the range of visible light of the electromagnetic spectrum provides information of ocean color that is associated with the water constituents. This measurements are used to monitor the level of biological activity and the presence of particles in the water. Ocean features such as the concentration of chlorophyll, suspended sediment concentration and sea surface temperature have a significant impact on the dynamics of the ocean. The concentration of chlorophyll (chla), active pigments of phytoplankton photosynthesis, as a key indicator applied for assessment of water quality and biochemistry. Experimental algorithms chla related to internal communication various optical components in the water that may be change in space and time in the water with different optical characteristics. Therefore, the algorithms have been developed for one area may not work for other places and each region according to its specific characteristics needs that determined by an algorithm may be appropriate to local. We have tried treatment several algorithms for determination of chlorophyll, including experimental algorithms with a simple band ratio of blue-green band (i.e. OCx) and algorithms includes two bands ratio with variable $R_{r s}\left(\lambda_{2}\right) / R_{r s}\left(\lambda_{1}\right)$, the three bands ratio with variable $\left[R_{r s}\left(\lambda_{1}\right)^{-1}-R_{r s}\left(\lambda_{2}\right)^{-1}\right] \times R_{r s}\left(\lambda_{3}\right)$ and four bands ratio with variable $\left[R_{r s}\left(\lambda_{1}\right)^{-1}-R_{r s}\left(\lambda_{2}\right)^{-1}\right] /\left[R_{r s}\left(\lambda_{4}\right)^{-1}-R_{r s}\left(\lambda_{3}\right)^{-1}\right]$ that desired wavelength (i.e. $\lambda_{1}, \lambda_{2}, \lambda_{3}$ and $\lambda_{4}$ ) in the range of red and near-infrared wavelengths of the electromagnetic spectrum are in the region of the Persian Gulf and Oman Sea look. Despite the high importance of the Persian Gulf and Oman Sea which can have up basin countries, to now few studies have been done in this area. The focus of this article on the northern part of Oman Sea and Persian Gulf, the shores of neighboring Iran (case 2 water). In this paper, by using Landsat 8 satellite imageries, we have discussed chla concentrations and customizing different OC algorithms for this new dataset (Landsat-8 imagery). This satellite was launched in 2013 and its data using two sensors continuously are provided operating one sensor imager land (OLI: Operational Land Imager) and the Thermal Infrared Sensor (TIRS: Thermal InfraRed Sensor) and are available. This sensors collect image data, respectively, for the nine-band short wavelength in the range of 433-2300 $\mathrm{nm}$ and dual-band long wavelength thermal. Seven band of the nine band picked up by the sensor information of OLI to deal with sensors TM (Thematic Mapper) and ETM+ (Enhanced Thematic Mapper Plus) in previous satellite Landsat compatible and two other band, the band of coastal water (433 to $453 \mathrm{~nm}$ ) and Cirrus band (1360 to 1390 $\mathrm{nm}$ ), short wave infrared provides to measure water quality and high thin clouds. Since OLI sensor in Landsat satellite 8 compared with other sensors to study OC have been allocated a much better spatial resolution can be more accurate to determine changes in OC. To evaluate the results of the image sensor MODIS (Moderate Resolution Imaging Spectroradiometer) at the same time satellite images Landsat 8 is used. The statistical parameters used in order to evaluate the performance of different algorithms, including root mean square error (RMSE) and coefficient of determination $\left(\mathrm{R}^{2}\right)$, and on the basis of these parameters we choose the most appropriate algorithm for the area. Extracted results for implementing different OC algorithms clearly shows superiority of utilized method by $\mathrm{R}^{2}=0.71$ and $\mathrm{RMSE}=0.07$.
\end{abstract}

\section{INTRODUCTION}

Oceans and seas almost 70 percent of the Earth's surface and play an important role in the cycling of elements such as nitrogen, carbon, oxygen and sulfur[1]. Phytoplankton, microscopic organisms basis of the marine food chain, are responsible for most primary production in the ocean and there are everywhere in the ocean surface. Monitoring changes of phytoplankton in a very large extent from ocean is possible only through satellite measurements. Chlorophyll (chla), the photosynthetically active pigment of phytoplankton, and is soluble in water and produced yellow-green color. In addition to the light-absorbing, chla involved in back light with complex interactions[2].

Chlorophyll concentration as one of the main phytoplankton as representative for the conditions used chemical water quality and ecological status. So we know the exact time and place of chlorophyll can help to understand the water quality and will be useful for water management[3].
Satellite sensors can be used as a convenient tool to evaluate some parameters of water quality parameters that affect its reflectance spectrum, because they produce comprehensive maps from the area. Bio-optical algorithms have been developed to recover chla to describe radiance measurements of ocean. Waterleaving radianceat different wavelengths are converted to reflection and then are used in various algorithms.

For waters with high concentrations of phytoplankton populations, algorithm based on the red and near-infrared bands for retrieval chla concentrations are more effective[4]. Experimental algorithms that use the band ratio blue-green are designed for open water ocean (Case 1) and this algorithms is often designed for coastal waters, as the second type water (Case 2), where other optical components except phytoplankton such as the suspended sediments and that non-correlation with phytoplankton, are also present, do not performance[4].

The reflection of the suspended sediments increases in nearinfrared band, so simple band ratio algorithms cannot discern 
high chla concentration and high sediment concentrations. In these cases, algorithms based on the ratio of red and near-infrared bands are most successful in predicting the concentration chla[5]. Activity ocean color studies began (1970) with sensor CZCS and expanded with sensors such as MODIS, SeaWIFS.

Chengfeng Le et al in 2013, the use of algorithms, dual-band, triband and quad-band (described in detail in the second part) in Florida using field data nine ship during 1998 to 2010 with MODIS and MERIS satellite data and concluded that the algorithms used in areas with chlorophyll concentrations greater than 4 mgm- 3 with a relative error of less than $30 \%$ is offered promising results[5].

Vasily et al in 2014, study the concentration of chlorophyll in the Gulf of Finland and Using algorithms OC3 and OC4 using MODIS satellite data and field data for the years 2012 and 2013 and Since the two algorithms overstate the estimate the concentrations of chla they have developed a new algorithm for the area that have good compatibility with field data[6].

Sun et al in 2014, assessment the dual-band and tri-band and quad-band algorithms in the mouth of two rivers in America using field data and MODIS data and concluded that the two band algorithm had better performance than three band and four band algorithms[3].

Tebbs et al in 2013, study the using Landsat+ ETM data linear relationship between chlorophyll concentration and remote sensing reflectance $(R r s)$ at a wavelength of $835 \mathrm{~nm}$, and tested the relationship between chlorophyll concentration and the ratio of two wavelength band between 835 and 660 and found that the algorithms based on the ratio of red and near-infrared bands are most successful in predicting chlorophyll concentration [4].

According to the literature in the field, extracting physical parameters color of the sea, especially its concentration (chla) and studies, shows the low number of methods for second type of water (coastal waters). However, with the launch of a new satellite optics with high spatial and spectral resolution is possible that this could be the physical parameters of sea and ocean in areas close to the coast to be modeled using this data.

In this paper, Landsat 8 data with spatial resolution much better than previous ocean color sensors to determine the amount of chlorophyll in coastal areas and compare the results with chlorophyll concentrations provided by the sensors with spatial resolution medium and low.

\section{OCEAN COLOR ALGORITHMS}

In this section, different bio-optical OC algorithms for retrieving chla and explaining ocean radiance measurements have been discussed. Also, in this section formulation of OC algorithms for calculating the chlorophyll are introduced.

\subsection{Simple band ratio algorithms (OCx algorithms)}

Simple band ratio algorithms are based on empirical regression between blue and green bands and chla. The correlation between the ratio of blue to green and chla is discussed in this kind of algorithms. The algorithms provide valuable results for open ocean waters that they are simple optically. This algorithm is often in the form of Eq 1.

$$
c=10^{\sum_{i=0}^{n} a_{i} x^{i}}, \quad x=\frac{\operatorname{Rrs}(\lambda \text { blue })}{\operatorname{Rrs}(\lambda \text { green })}
$$

Here $C$ represents the concentration of chlorophyll and the Rrs is reflection remote sensing in wavelength $\lambda[4]$.

\subsection{The algorithm based on the band ratio of RED and NIR}

In this algorithm, the ratio of red to near-infrared band used to extract the correlation chla in three modes (two, three and four bands) [5].

\subsubsection{Two-band approach}

This algorithm is based on the principle that it is positioning the red band $\left(\lambda_{1}\right)$ located in the vicinity of the maximum absorption chla while the near-infrared band $\left(\lambda_{2}\right)$ in a region that is absorbed by pigments of phytoplankton. Also, soluble colored organic matter (CDOM) is lowest, and dual-band index is an indicator of chla in some areas [5]. This algorithm is often in the form of Eq 2.

$$
C=a x^{b}, x=\operatorname{Rrs}\left(\lambda_{2}\right) \times \operatorname{Rrs}\left(\lambda_{1}\right)^{-1}
$$

Where $C$ represents the concentration of chlorophyll, $a$ and $b$ are constants that must estimate and Rrs is reflection remote sensing.

\subsubsection{Three-band approach}

This algorithm is based on three main assumptions: (1) CDOM and detrital absorptions at $\lambda_{2}$ are close to those at $\lambda_{1} ;(2) \operatorname{Rrs}\left(\lambda_{3}\right)$ is influenced by backscattering only, with minimal influence from water constituent absorption; and (3) backscattering is approximately equal at the three wavelengths. This algorithm has been used successfully in several coastal water [5]. This algorithm is often in the form of Eq 3.

$$
c=a e^{b x}, x=\left[\operatorname{Rrs}\left(\lambda_{1}\right)^{-1}-\operatorname{Rrs}\left(\lambda_{2}\right)^{-1}\right] \times \operatorname{Rrs}\left(\lambda_{3}\right)
$$

Where $C$ represents the concentration of chlorophyll, $a$ and $b$ are constants that must estimate and Rrs is reflection remote sensing.

\subsubsection{The four-band approach}

This algorithm is similar to the three-band but with an additional band which is applied when absorbed water components $\lambda_{3}$ negligible enough not. According to the form algorithm requires two additional assumptions:

1) CDOM and detrital absorptions at $\lambda_{2}$ are close to those at $\lambda_{1}$ and the same is true for $\lambda_{4}$ and $\lambda_{3}$.

2) backscattering is independent of wavelength[5]. This algorithm is often in the form of Eq 4.

$$
\begin{gathered}
c=a x+b, \\
x=\left[\operatorname{Rrs}\left(\lambda_{1}\right)^{-1}-\operatorname{Rrs}\left(\lambda_{2}\right)^{-1}\right] \times\left[\operatorname{Rrs}\left(\lambda_{4}\right)^{-1}-\operatorname{Rrs}\left(\lambda_{3}\right)^{-1}\right]^{-1}
\end{gathered}
$$

Where $\mathrm{C}$ represents the concentration of chlorophyll, $a$ and $b$ are constants that must estimate and $\operatorname{Rrs}$ is reflection remote sensing[4].

\section{STUDY AREA AND DATA SETS}

This section have paid to study area and satellite images data sets used to estimate the chlorophyll concentration using different OC algorithms.

\subsection{Image data sets}

Landsat 8 satellite was launched in 2013 and it continually has been prepared and made available two sensor data, including Operational Land Imager (OLI) and the Thermal Infrared Sensor (TIRS). This two sensors collect image data respectively for the nine-band and dual-band wavelength in the range of 433-2300 $\mathrm{nm}$ long wavelength thermal. OLI has two new bands in addition to the legacy Landsat bands (1-5, 7, and Pan). Two other band, the Blue Coast band (433-453 nm) and Cirrus band (1360-1390 $\mathrm{nm})$ short wave infrared can measure water quality and provides high thin clouds[7].

Since OLI sensor on Landsat 8 compared with other sensors that have been assigned to study OC is much better spatial resolution, it can accurately determine the changes of the OC. Landsat 8 satellite images used and downloaded in this article from the site [8]. The date of incorporated images are October 25, 2014, November 10, 2014, and December 3, 2014. Also, to evaluate the 
results, the level 2 data set of MODIS sensor on the Aqua satellite with different spatial resolution is used and download from [9].

\subsection{Study area}

Study area includes the Persian Gulf and Hormuz Strait, which is located in the North West Indian Ocean. Persian Gulf is a shallow, semi-enclosed waters with an average depth of $36 \mathrm{~m}$ and maximum depth of between 90 and 800 meters in the northeast and is located within Iranian waters, its maximum depth is in the Hormuz Strait[10]. This paper will focus on the northern part of the Persian Gulf Iran (Figure 1).

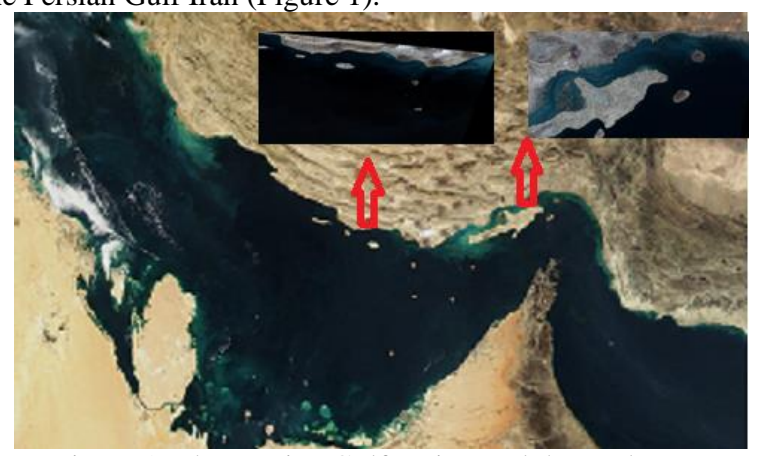

Figure 1. The Persian Gulf region and the study area 4. PROPOSED METHOD

Figure 2 shows the different stages of the proposed algorithm.

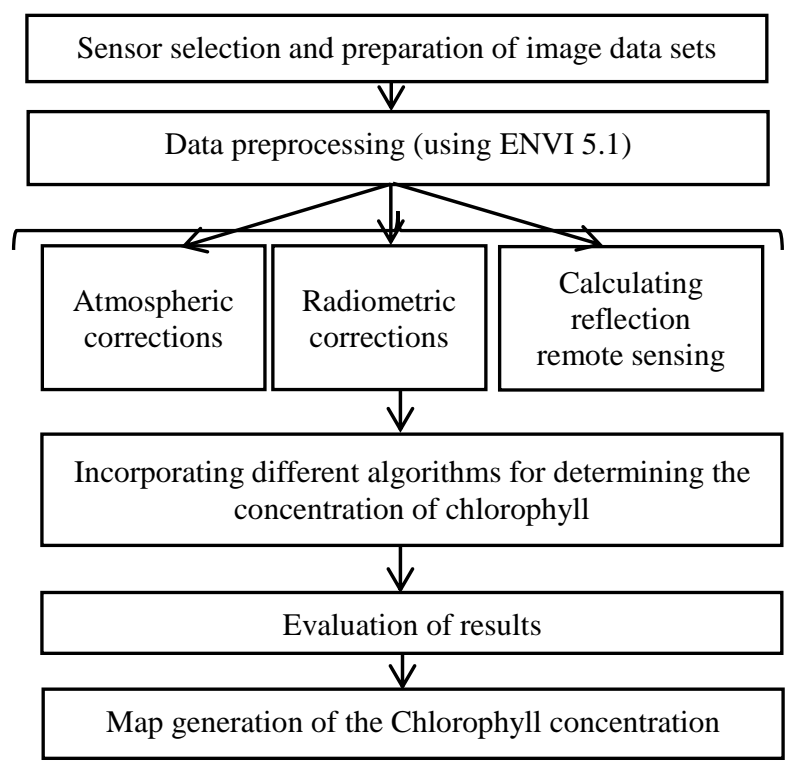

Figure 2. Different stages of selecting the best algorithm Data of Landsat 8 were downloaded and processed using ENVI 5.1 software. Also, atmospheric correction is performed by FLAASH for each image data set. The output of this stage Rrs corrected for each pixel in bands 1 to 7 , which it values is applied at each of the algorithms in this paper (i.e. OC2, OC3, OC4, twoband approach and three-band approach algorithms).

The estimated coefficients for each algorithm are performed using least square method. The output and performance result of each algorithm evaluated using statistical values of RMSE and $\mathrm{R}^{2}$. Finally, the most appropriate algorithm will be selected (with regard to errors).

\section{EXPERIMENT AND RESULTS}

The statistical parameters and criteria is used to evaluate the performance of different OC algorithms, including RMSE and $\mathrm{R}^{2}$ is based on the results of these parameters, selection of the most

appropriate algorithm is possible. The brief implemented OC algorithms that are incorporated in this study is illustrated in Table 1.

Table 1. Different OC algorithm customized in this study.

\begin{tabular}{|c|c|}
\hline By & Ocean Color Algorithm $($ OCA $)$ \\
\hline$[4]$ & chla $=a x^{b}, \quad x=R_{r s}\left(\lambda_{2}\right) / R_{r s}\left(\lambda_{1}\right)$ \\
\hline$[4]$ & chla $=a x^{b}, x=\left[R_{r s}\left(\lambda_{1}\right)^{-1}-R_{r s}\left(\lambda_{2}\right)^{-1}\right] \times R_{r s}\left(\lambda_{3}\right)$ \\
\hline$[4]$ & chla $=a x+b, x=\left[R_{r s}\left(\lambda_{1}\right)^{-1}-R_{r s}\left(\lambda_{2}\right)^{-1}\right] /\left[R_{r s}\left(\lambda_{4}\right)^{-1}\right.$ \\
\hline$[8],[9$, & $\left.-R_{r s}\left(\lambda_{3}\right)^{-1}\right]$ \\
p. 4] & $x=\operatorname{cog}_{10}\left\{\left[R_{r s}\left(\lambda_{1}\right)>R_{r s}\left(\lambda_{2}\right)\right] / R_{r s}\left(\lambda_{3}\right)\right\}$ \\
\hline
\end{tabular}

Table 2 shows the results and performances provided by these different $\mathrm{OC}$ algorithms. The residuals and remaining charts for each algorithm is showed in Figure 3. By comparing the statistical parameters form Table 2, it is clear that two band and three band algorithms have the lowest performance. Also, performance of the OC2, OC3 and OC4 algorithms have good degree of consistency comparing to other algorithms. As it is clear from Table 2, OC2 and OC 3 number of observations and inputs more than others methods and this is related to the equation of each algorithm. For avoiding zero values, the denominator or the fraction of the infinite many two band and three band observations, is not used in computations. The residuals and remains graph are showed in Figure 3 for better understanding of utilized OC algorithms. The remaining graph two-band and three-band algorithms are sparse while the remaining graph of OC4 and OC2 and OC3 algorithms are concentrated around the zero point.

Table 2. Estimated RMSE and $\mathrm{R}^{2}$ values for different algorithms and related coefficient.

\begin{tabular}{|c|c|c|c|c|c|}
\hline & Oc2 & Oc3 & Oc4 & two band & $\begin{array}{l}\text { three } \\
\text { band }\end{array}$ \\
\hline 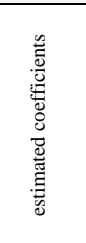 & $\begin{array}{c}\text { b1 } \\
0.29201 \\
\text { b2 } \\
1.9985 \\
\text { b3 } \\
1.7639 \\
\text { b4 } \\
-6.2326\end{array}$ & $\begin{array}{c}\text { b1 } \\
0.30354 \\
\text { b2 } \\
0.4481 \\
\text { b3 } \\
-28.909 \\
\text { b4 } \\
-154.47 \\
\text { b5 } \\
-207.77\end{array}$ & $\begin{array}{c}\text { b1 } \\
-0.54731 \\
\text { b2 } \\
-3.8617 \\
\text { b3 } \\
-6.4984 \\
\text { b4 } \\
-4.1701 \\
\text { b5 } \\
-\mathbf{- 0 . 9 0 5 6 6}\end{array}$ & $\begin{array}{c}\text { b1 } \\
1.5329 \\
\text { b2 } \\
-\mathbf{0 . 0 3 6 3 1}\end{array}$ & $\begin{array}{c}\text { b1 } \\
0.33387 \\
\text { b2 } \\
-\mathbf{0 . 0 0 4 4}\end{array}$ \\
\hline \# inputs & 62997 & 62353 & 46665 & 32952 & 32923 \\
\hline RMSE & 0.096 & 0.0776 & 0.103 & 0.529 & 0.398 \\
\hline $\mathrm{R}^{2}$ & 0.558 & 0.712 & 0.533 & 0.0045 & 0.0109 \\
\hline
\end{tabular}
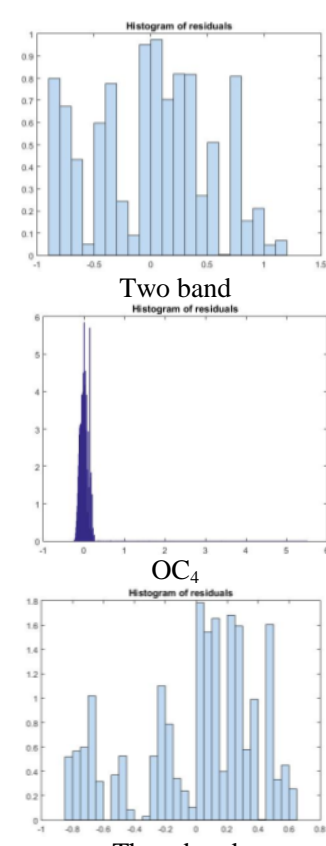

Three band

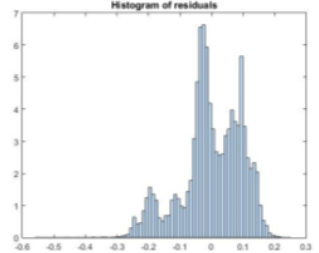

$\mathrm{OC}_{2}$

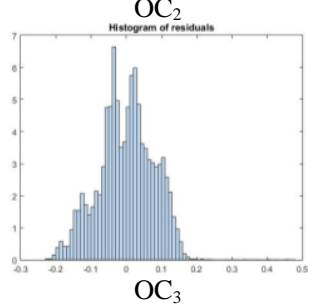

Figure 3. The remaining graph of different algorithms. 
In Figure 4, RMSE and $\mathrm{R}^{2}$ values for different algorithms to compare more easily displayed on charts.

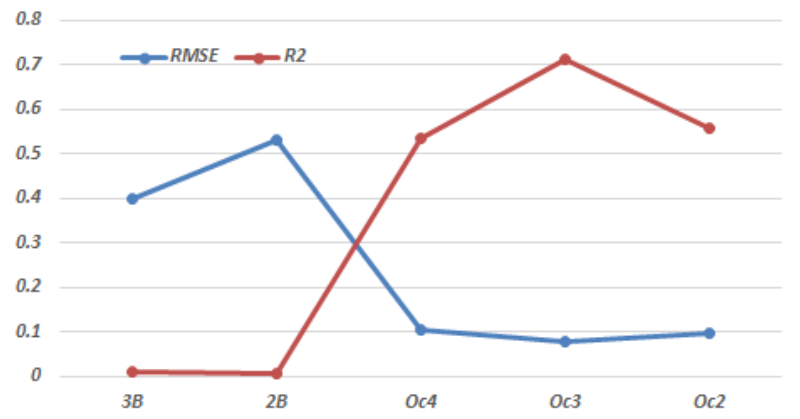

Figure 4. Comparison of performance of different algorithms As it clear form OC3 algorithm, the largest amount of $\mathrm{R}^{2}(0.713)$ and the lowest RMSE (0.077) is the most appropriate algorithm in the desired area. Finally, new coefficients for algorithm using Landsat8 data sets for study area are achieved (Eq 5).

$$
\begin{gathered}
C=10^{(a 0+a l x+a 2 x 2+a 3 \times 3+a 4 \times 4)} \\
\boldsymbol{x}=\log \frac{\max \{\operatorname{Rrs}(\mathbf{0 . 4 4 3}) \operatorname{or} \boldsymbol{R r s}(\mathbf{0 . 4 8 3})\}}{\operatorname{Rrs}(\mathbf{0 . 5 6 1})}
\end{gathered}
$$

The coefficients for Eq 5 are $\mathrm{a}_{0}, \mathrm{a}_{1}, \mathrm{a}_{2}, \mathrm{a}_{3}$ and $\mathrm{a}_{4}$ 0.30354, 0.4481, $-28.909,-154.47$ and -207.77 , respectively. In Figure 5 Chlorophyll values determined using the most appropriate algorithm is shown in part of the Persian Gulf.

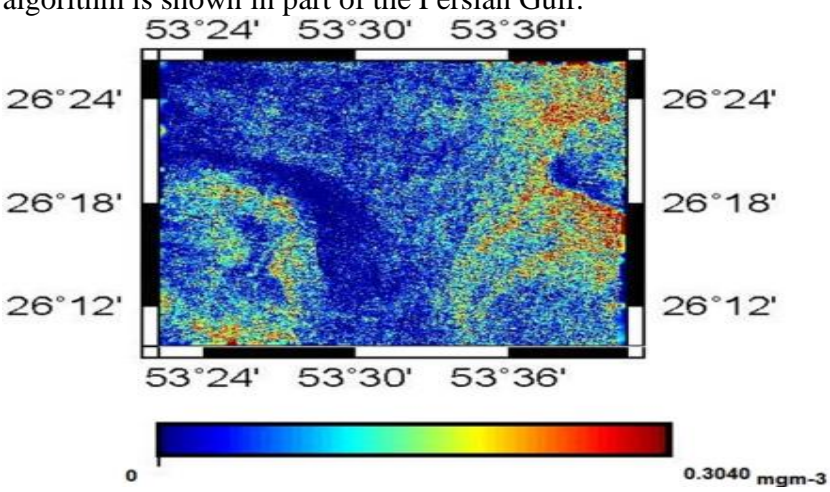

Figure 5. Estimated chlorophyll concentration using Landsat-8 imagery.

\section{CONCLUSIONS}

In this paper, according to implemented procedures, the performance of five OC algorithms for estimating the chlorophyll concentration using Landsat 8 imagery were evaluated in the Persian Gulf. Also, in this area the most compatible and appropriate algorithm is chosen base on the proposed algorithms to determine the chlorophyll concentration in areas near the coast. On the other hands, due to much better spatial resolution of Landsat8 imagery, estimated chlorophyll concentration has more detail for investigating and future studies. Despite good spatial resolution of Landsat 8 imagery, its temporal resolution (every 16 days) is low and this prevents the observation of processes with short cycle and this is one of the issues that must be resolved through a combination of several sensor.

\section{REFERENCES}

[1] S. Dey and R. P. Singh, "Comparison of chlorophyll distributions in the northeastern Arabian Sea and southern Bay of Bengal using IRS-P4 Ocean Color Monitor data," Remote Sens. Environ., vol. 85, no. 4, pp. 424-428, Jun. 2003.
[2] R. A. Shuchman, G. Leshkevich, M. J. Sayers, T. H. Johengen, C. N. Brooks, and D. Pozdnyakov, "An algorithm to retrieve chlorophyll, dissolved organic carbon, and suspended minerals from Great Lakes satellite data," $J$. Gt. Lakes Res., vol. 39, Supplement 1, pp. 14-33, 2013.

[3] D. Sun, C. Hu, Z. Qiu, J. P. Cannizzaro, and B. B. Barnes, "Influence of a red band-based water classification approach on chlorophyll algorithms for optically complex estuaries," Remote Sens. Environ., vol. 155, pp. 289-302, Dec. 2014.

[4] E. J. Tebbs, J. J. Remedios, and D. M. Harper, "Remote sensing of chlorophyll-a as a measure of cyanobacterial biomass in Lake Bogoria, a hypertrophic, saline-alkaline, flamingo lake, using Landsat ETM +," Remote Sens. Environ., vol. 135, pp. 92-106, Aug. 2013.

[5] C. Le, C. Hu, J. Cannizzaro, D. English, F. Muller-Karger, and Z. Lee, "Evaluation of chlorophyll-a remote sensing algorithms for an optically complex estuary," Remote Sens. Environ., vol. 129, pp. 75-89, Feb. 2013.

[6] S. Vazyulya, A. Khrapko, O. Kopelevich, V. Burenkov, T. Eremina, and A. Isaev, "Regional algorithms for the estimation of chlorophyll and suspended matter concentration in the Gulf of Finland from MODIS-Aqua satellite data*," Oceanologia, vol. 56, no. 4, pp. 737-756, 2014.

[7] "Landsat8DataUsersHandbook.pdf." .

[8] "EarthExplorer." [Online]. Available: http://earthexplorer.usgs.gov/. [Accessed: 18-Dec-2015].

[9] "OceanColor Home Page | NASA Ocean Color." [Online]. Available: http://oceancolor.gsfc.nasa.gov/cms/. [Accessed: 18-Dec-2015].

[10] D. Dadyzadh and H. Malakoti, "dust storms and their ecological effects on the distribution of chlorophyll in the Gulf," Journal of Aquatic Ecology, vol. 4, no. 3, pp. 82-73, Jan. 2015. 